\title{
An alternative perspective to observe the critical phenomena of dilaton black holes
}

\author{
Jie-Xiong $\mathrm{Mo}^{\mathrm{a}}$ \\ Institute of Theoretical Physics, Lingnan Normal University, Zhanjiang 524048, Guangdong, China
}

Received: 7 June 2017 / Accepted: 22 July 2017 / Published online: 9 August 2017

(C) The Author(s) 2017. This article is an open access publication

\begin{abstract}
The critical phenomena of dilaton black holes are probed from a totally different perspective other than the $P-v$ criticality and the $q-U$ criticality discussed in former literature. We investigate not only the two point correlation function but also the entanglement entropy of dilaton black holes. For both the two point correlation function and the entanglement entropy we consider $4 \times 2 \times 2=16$ cases due to different choices of parameters. The van der Waals-like behavior can be clearly witnessed from all the $T-\delta L(T-\delta S)$ graphs for $q<q_{c}$. Moreover, the effects of dilaton gravity and the spacetime dimensionality on the phase structure of dilaton black holes are disclosed. Furthermore, we discuss the stability of dilaton black holes by applying the analogous specific heat definition and remove the unstable branch by introducing a bar $T=T_{*}$. It is shown that the first order phase transition temperature $T_{*}$ is affected by both $\alpha$ and $n$. The analogous equal area laws for both the $T-\delta L$ graph and the $T-\delta S$ graph are examined numerically. The relative errors for all cases are small enough so that we can safely conclude that the analogous equal area laws hold for $T-\delta L$ $(T-\delta S)$ graph of dilaton black holes.
\end{abstract}

\section{Introduction}

Recently Johnson disclosed intriguing properties of entanglement entropy [1]. It was shown that the isocharges in the entanglement entropy-temperature plane and those of the entropy-temperature plane not only enjoy similar behavior but also share the same critical temperature and critical exponents [1]. Moreover, Ref. [2] proved that the equal area law which in former literature [3] was shown to hold for the $T-S$ curve, also holds for the entanglement entropy-temperature plane. Reference [4] further showed that other nonlocal observable, such as the two point correlation function, also display similar behavior as the entanglement entropy. The

a e-mail: mojiexiong@gmail.com research topic initiated by Johnson is receiving more and more attention [5-12].

In this paper we would like to generalize the above topic to dilaton black holes in order to probe whether these phenomena are universal. On the other hand, our probe may be served as an alternative perspective to observe the critical phenomena of dilaton black holes and would help to deepen the understanding of these phenomena. Investigating the properties of black holes in dilaton gravity is of interest in itself. The dilaton field appears in the low energy limit of string theory and has significant impact on both the casual structure and thermodynamics of black holes. The action of dilaton gravity contains one or more Liouville-type potentials. These potentials are resulted by the breaking of spacetime supersymmetry in ten dimensions. Both the black hole solutions in dilaton gravity and their thermodynamics have attracted considerable attention [13-49].

The paper is organized as follows. Section 2 is devoted to a brief review of thermodynamics of dilaton black holes. In Sect. 3 their two point correlation functions are studied numerically. We will carry out a numerical check of the equal area law in Sect. 4. Furthermore, we will investigate their entanglement entropy numerically in Sect. 5 and check the corresponding equal area law in Sect. 6. The last section contains conclusions.

\section{A brief review of thermodynamics of dilaton black holes}

The $(n+1)$-dimensional Einstein-Maxwell-dilaton action was reviewed in Ref. [35] as

$$
\begin{aligned}
S= & \frac{1}{16 \pi} \int d^{n+1} x \sqrt{-g}\left(R-\frac{4}{n-1}(\nabla \Phi)^{2}\right. \\
& \left.-V(\Phi)-e^{-4 \alpha \Phi /(n-1)} F_{\mu \nu} F^{\mu \nu}\right),
\end{aligned}
$$




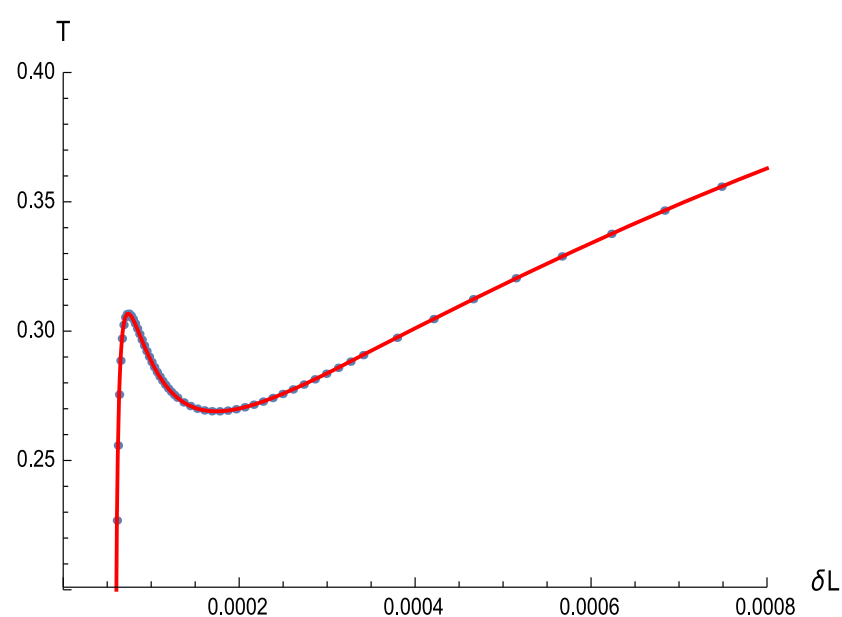

(a)

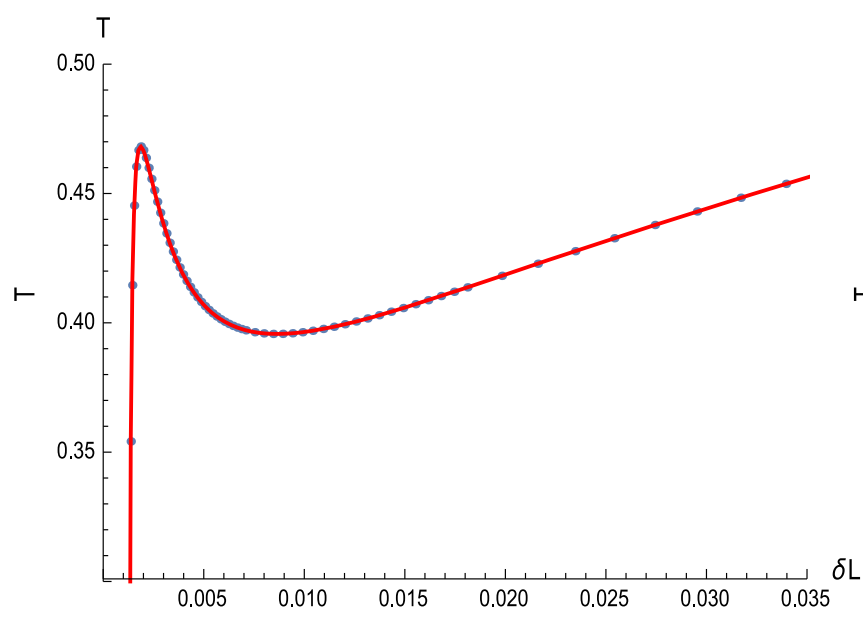

(c)

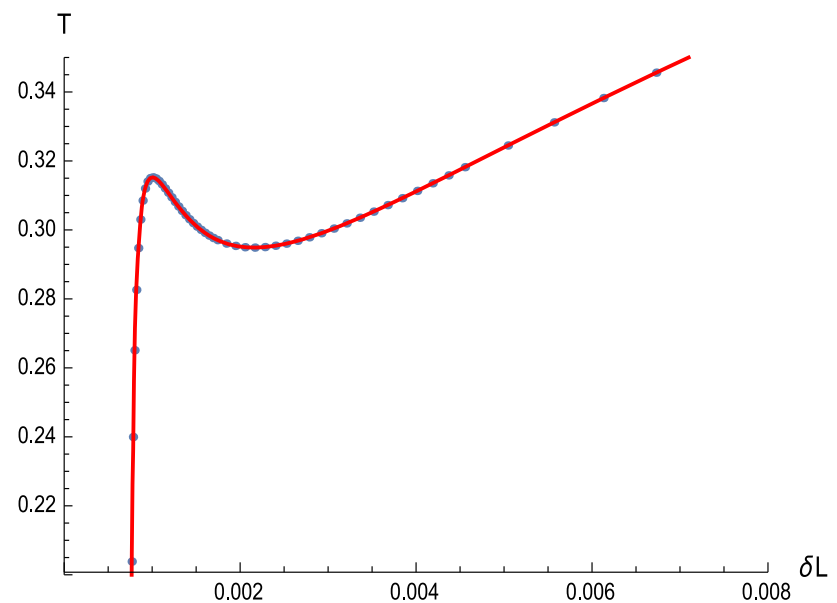

(b)

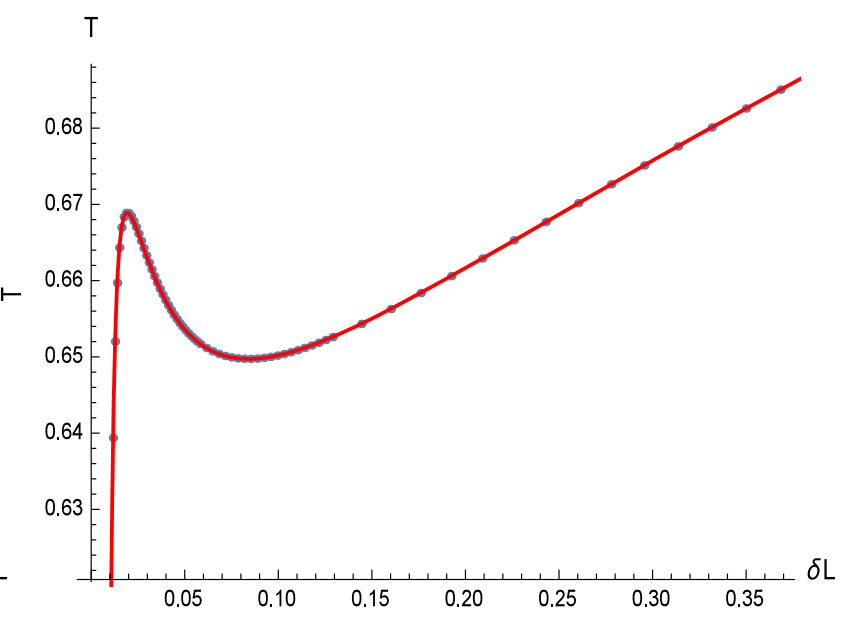

(d)

Fig. $1 T$ vs. $\delta L$ for $n=3, \theta_{0}=0.2$. a $\alpha=0, q=0.12, \mathbf{b} \alpha=0.25, q=0.12$, $\mathbf{c} \alpha=0.5, q=0.06$, $\mathbf{d} \alpha=0.75, q=0.06$

where $\Phi$ is the dilaton field with its potential denoted as $V(\Phi) . R$ is the Ricci scalar curvature and $F_{\mu \nu}=\partial_{\mu} A_{\nu}-$ $\partial_{\nu} A_{\mu}$ is the electromagnetic field tensor. The strength of coupling between the electromagnetic field and the scalar field is characterized by $\alpha$.

The corresponding solution was reviewed in Ref. [35] as

$$
\begin{aligned}
& \mathrm{d} s^{2}=-f(r) \mathrm{d} t^{2}+\frac{1}{f(r)} \mathrm{d} r^{2}+r^{2} R^{2}(r) \mathrm{d} \Omega_{k, n-1}^{2}, \\
& \Phi(r)=\frac{(n-1) \alpha}{2\left(\alpha^{2}+1\right)} \ln \left(\frac{b}{r}\right),
\end{aligned}
$$

where

$$
\begin{aligned}
f(r)= & -\frac{k(n-2)\left(1+\alpha^{2}\right)^{2} b^{-2 \gamma} r^{2 \gamma}}{\left(\alpha^{2}-1\right)\left(\alpha^{2}+n-2\right)} \\
& -\frac{m}{r^{(n-1)(1-\gamma)-1}}-\frac{n b^{2 \gamma}\left(1+\alpha^{2}\right)^{2} r^{2(1-\gamma)}}{l^{2}\left(\alpha^{2}-n\right)} \\
& +\frac{2 q^{2}\left(1+\alpha^{2}\right)^{2} b^{-2(n-2) \gamma} r^{2(n-2)(\gamma-1)}}{(n-1)\left(n+\alpha^{2}-2\right)},
\end{aligned}
$$

$R(r)=e^{2 \alpha \Phi(r) /(n-1)}$.

$k$ is a constant characterizing the hypersurface $\mathrm{d} \Omega_{k, n-1}^{2}$ whose volume is denoted as $\omega_{n-1} . k$ respectively can be taken as $-1,0,1$, corresponding to the hyperbolic, flat and spherical constant curvature hypersurface. $\gamma$ is related to $\alpha$ by $\gamma=\alpha^{2} /\left(\alpha^{2}+1\right) . b$ is an arbitrary constant while $l$ is the AdS length scale. $q$ and $m$ are constants related to the charge $Q$ and the mass $M$ of the black hole as follows:

$M=\frac{b^{(n-1) \gamma}(n-1) \omega_{n-1} m}{16 \pi\left(\alpha^{2}+1\right)}$,

$Q=\frac{\omega_{n-1} q}{4 \pi}$.

Based on both the fact that the term including $m$ should vanish at spatial infinity and the fact that the electric potential $A_{t}$ should be finite at infinity, Ref. [43] obtained the restrictions on $\alpha$ for dilaton black holes coupled with power-law Maxwell field as follows: 


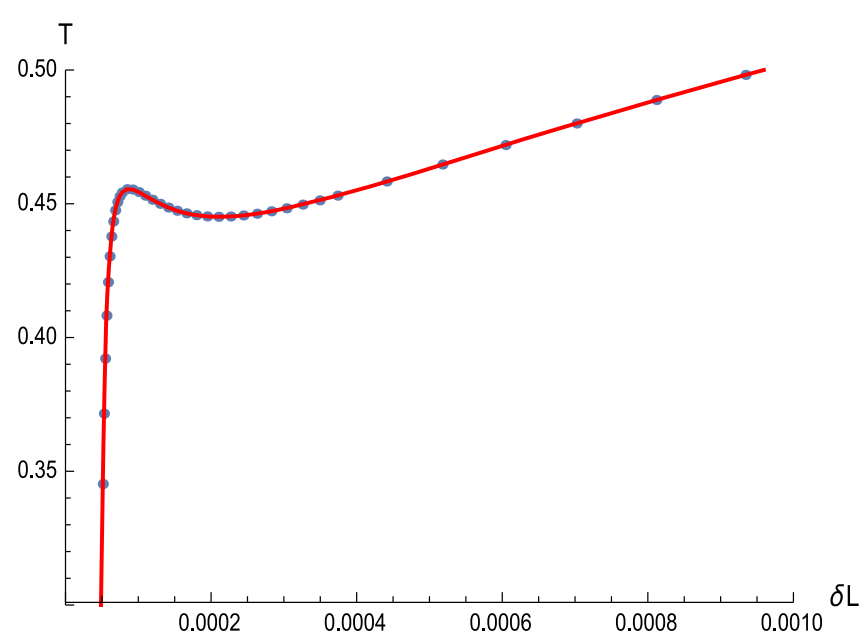

(a)

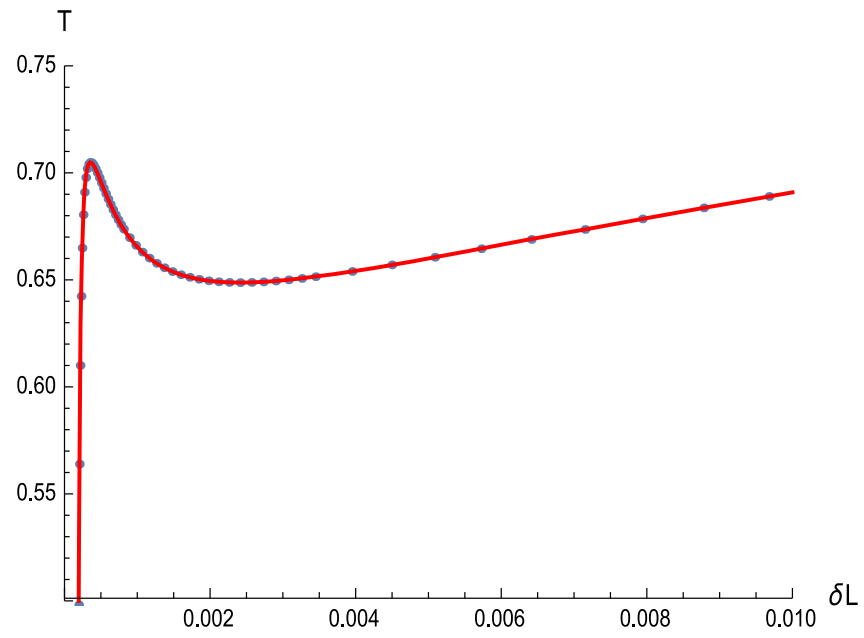

(c)

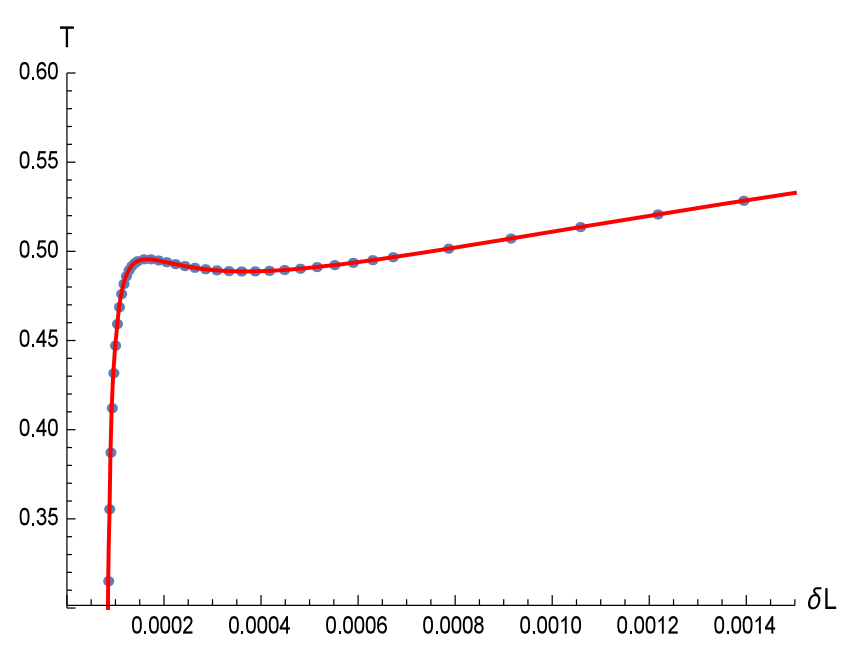

(b)

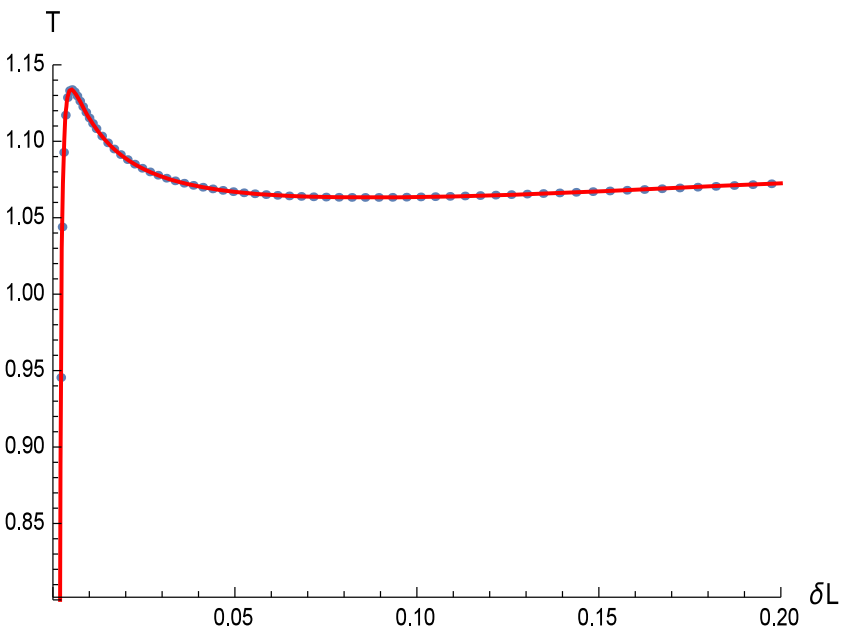

(d)

Fig. $2 T$ vs. $\delta L$ for $n=4, \theta_{0}=0.2$. $\mathbf{a} \alpha=0, q=0.12$, $\mathbf{b} \alpha=0.25, q=0.12, \mathbf{c} \alpha=0.5, q=0.06$, $\mathbf{d} \alpha=0.75, q=0.06$

For $\frac{1}{2}<p<\frac{n}{2}, \quad 0 \leq \alpha^{2}<n-2$,

For $\frac{n}{2}<p<n-1, \quad 2 p-n<\alpha^{2}<n-2$.

In this paper we consider the dilaton black holes coupled with a standard Maxwell field, corresponding to the case $p=1$. So the above restriction is reduced to $0 \leq \alpha^{2}<n-2$.

The Hawking temperature and the entropy have been derived as [35]

$$
\begin{aligned}
T= & \frac{-\left(1+\alpha^{2}\right)}{2 \pi(n-1)}\left[\frac{k(n-2)(n-1)}{2 b^{2 \gamma}\left(\alpha^{2}-1\right)} r_{+}^{2 \gamma-1}\right. \\
& \left.+\Lambda b^{2 \gamma} r_{+}^{1-2 \gamma}+q^{2} b^{-2(n-2) \gamma} r_{+}^{(2 n-3)(\gamma-1)-\gamma}\right] \\
S= & \frac{\omega_{n-1} b^{(n-1) \gamma} r_{+}^{(n-1)(1-\gamma)}}{4},
\end{aligned}
$$

where the relation $\Lambda=-n(n-1) / 2 l^{2}$ holds for $(n+1)$ dimensional AdS black holes.

Furthermore, Ref. [35] investigated the $P-v$ criticality of dilaton black holes when $Q$ was treated as an invariant parameter and the $q-U$ criticality ( $U$ denotes the electric potential) when $l$ was treated as an invariant parameter. Both cases were shown to exhibit van der Waals-like behavior [35]. In the rest of this paper we will further probe the critical phenomena of dilaton black holes from a totally different perspective. Namely, the two point correlation function and the entanglement entropy.

\section{Two point correlation function of dilaton black holes and its van der Waals like behavior}

Considering the points $\left(t_{0}, x_{i}\right)$ and $\left(t_{0}, x_{j}\right)$ on the boundary with the corresponding bulk geodesic length denoted as $L$, 


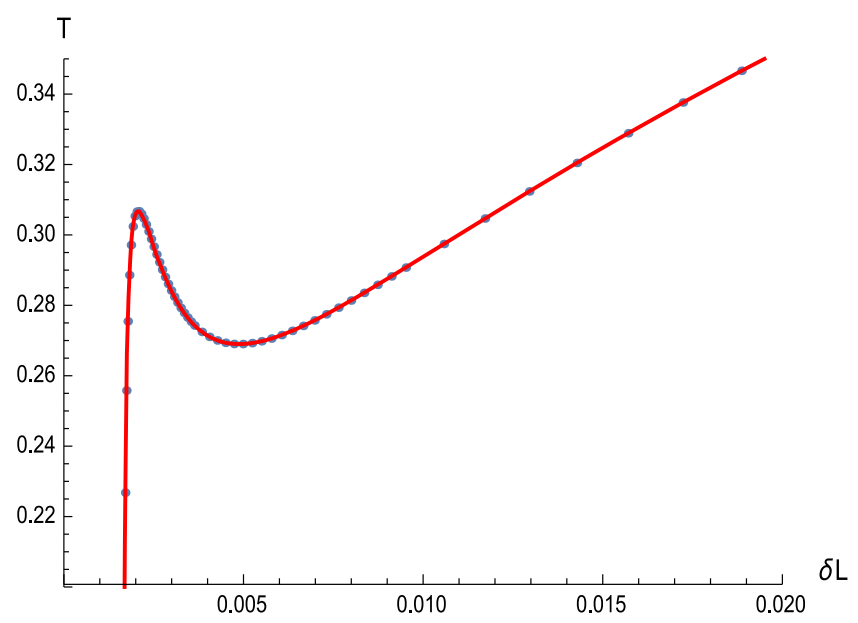

(a)

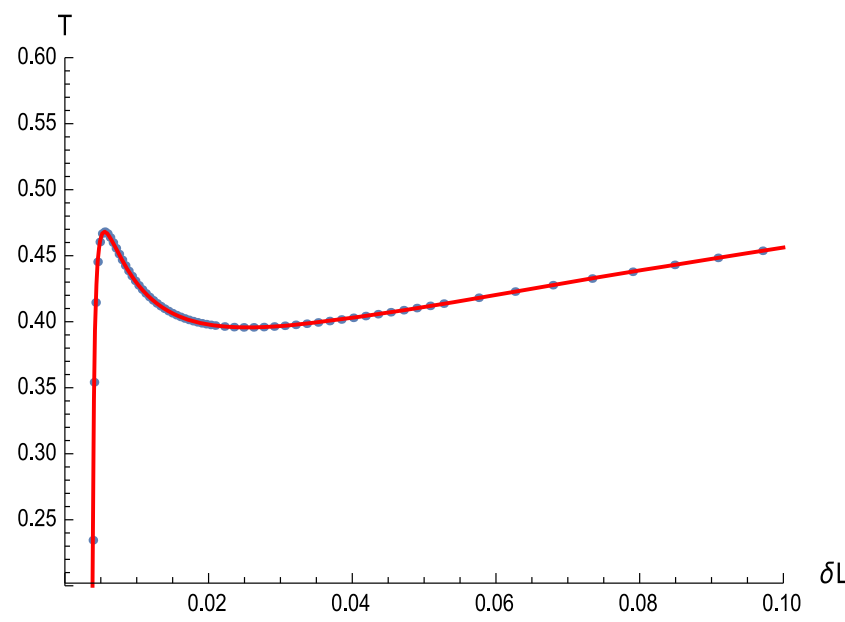

(c)

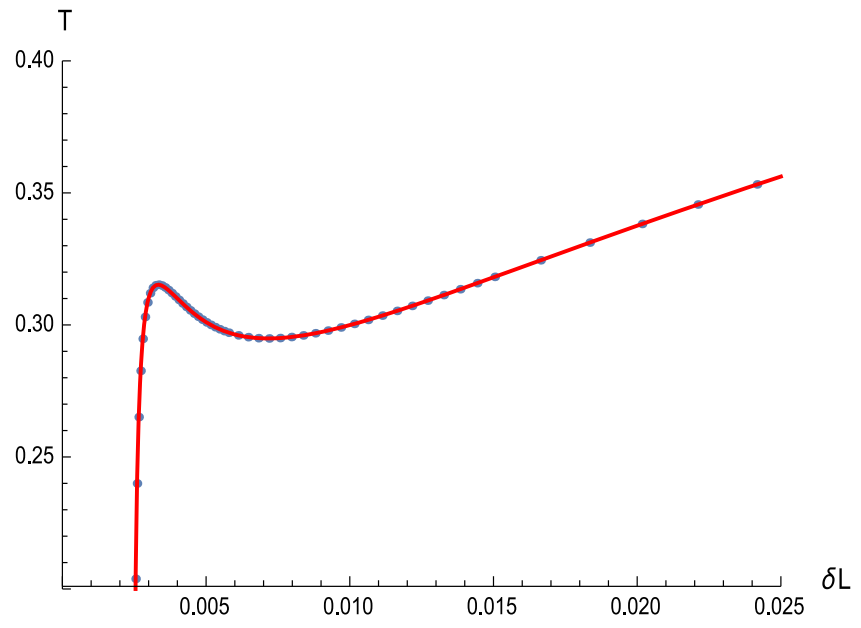

(b)

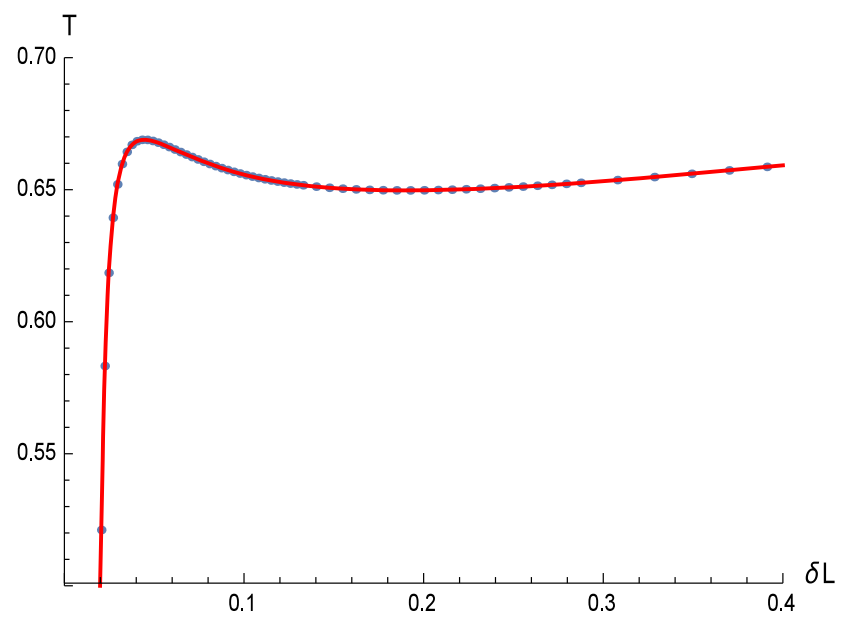

(d)

Fig. $3 T$ vs. $\delta L$ for $n=3, \theta_{0}=0.3$. $\mathbf{a} \alpha=0, q=0.12$, $\mathbf{b} \alpha=0.25, q=0.12, \mathbf{c} \alpha=0.5, q=0.06$, $\mathbf{d} \alpha=0.75, q=0.06$

the equal time two point correlation function in the large $\Delta$ limit ( $\Delta$ denotes the conformal dimension of scalar operator $\mathcal{O}$ in the dual field theory) takes the following form [50]:

$\left\langle\mathcal{O}\left(t_{0}, x_{i}\right) \mathcal{O}\left(t_{0}, x_{j}\right)\right\rangle \approx e^{-\Delta L}$.

The proper length can be obtained by parameterizing the trajectory with $\theta$

$L=\int_{0}^{\theta_{0}} \mathcal{L}(r(\theta), \theta) \mathrm{d} \theta, \quad \mathcal{L}=\sqrt{\frac{r^{\prime 2}}{f(r)}+r^{2}}$,

where $r^{\prime}=\mathrm{d} r / \mathrm{d} \theta$. Note that the boundary points have been chosen as $\left(\phi=\frac{\pi}{2}, \theta=0\right)$ and $\left(\phi=\frac{\pi}{2}, \theta=\theta_{0}\right)$.

Applying the well-known Euler-Lagrange equation

$\frac{\partial \mathcal{L}}{\partial r}=\frac{d}{\mathrm{~d} \theta}\left(\frac{\partial \mathcal{L}}{\partial r^{\prime}}\right)$ the equation of motion for $r(\theta)$ can be derived. Solving the equation of motion constrained by the boundary condition $r(0)=r_{0}, r^{\prime}(0)=0 r(\theta)$ is obtained by numerical methods. To avoid the divergence, the geodesic length $L_{0}$ in pure AdS with the same boundary region should be subtracted from the geodesic length $L$, with the regularized geodesic length denoted as $\delta L$. Note that $L_{0}$ can also be obtained through numerical treatment.

In order to investigate the effect of dilaton gravity on the phase structure of the two point correlation function, we focus on the cases where $\alpha$ respectively is chosen to be $0,0.25,0.5,0.75$. Note that we have considered the restriction $0 \leq \alpha^{2}<n-2$ mentioned in the former section.

On the other hand, $n$ respectively is chosen as 3,4 in order to study the possible effect of spacetime dimensionality. Moreover, we choose $\theta_{0}=0.2,0.3$ to check whether different boundary region sizes exert influence on the phase structure as has been shown in former literature [4]. In this 


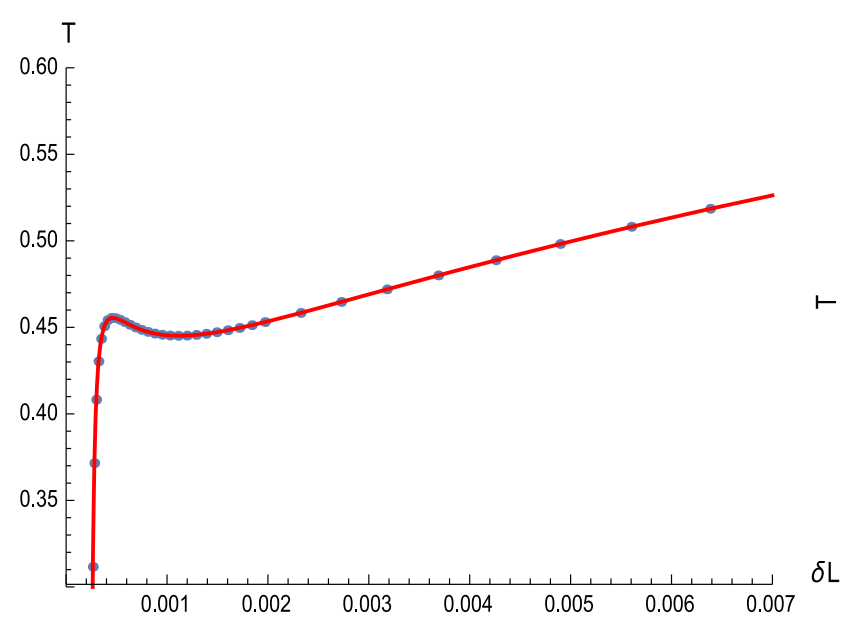

(a)

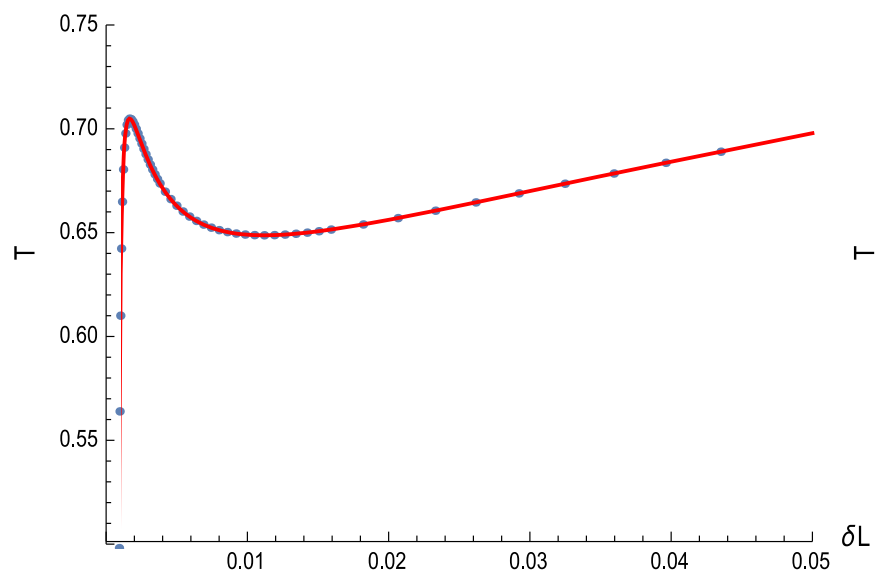

(c)

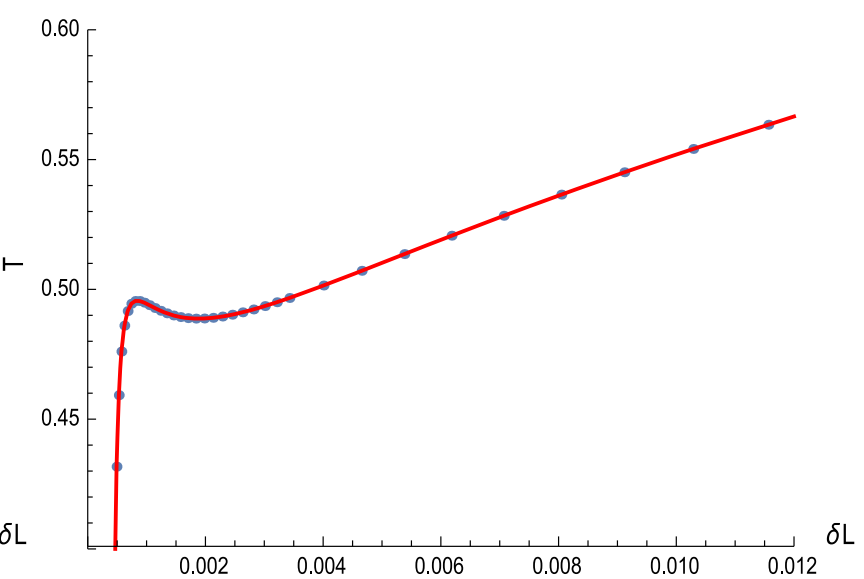

(b)

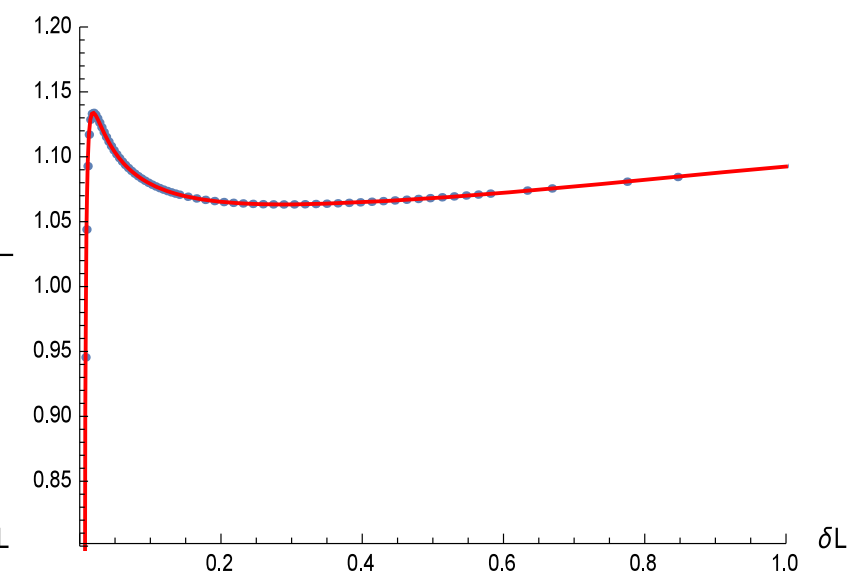

(d)

Fig. $4 T$ vs. $\delta L$ for $n=4, \theta_{0}=0.3$. a $\alpha=0, q=0.12, \mathbf{b} \alpha=0.25, q=0.12, \mathbf{c} \alpha=0.5, q=0.06$, $\mathbf{d} \alpha=0.75, q=0.06$

paper the cutoff $\theta_{c}$ accordingly will be chosen as $0.199,0.299$ and the AdS radius $l$ will be set to be one.

To summarize, we consider $4 \times 2 \times 2=16$ cases due to different choices of parameters. In each case we concentrate on the case $q<q_{c}$ ( $q_{c}$ denotes the critical value of the parameter $q$ ) to probe the possible van der Waals behavior in the $T-\delta L$ graphs. Figure $1 \mathrm{a}-\mathrm{d}$ respectively show the cases of $n=3, \theta_{0}=0.2$ with four different choices of $\alpha$ while Fig. 2a-d display the cases for $n=4, \theta_{0}=0.2$. The cases in which $n=3, \theta_{0}=0.3$ are depicted in Fig. 3a-d while the cases in which $n=4, \theta_{0}=0.3$ are depicted in Fig. 4a-b.

From all the $T-\delta L$ graphs for $q<q_{c}$, the van der Waalslike behavior can be clearly witnessed. There exist both the local maximum temperature and the local minimum temperature, which we respectively denote as $T_{\max }$ and $T_{\min }$. As shown in Figs. 1, 2, 3 and 4, the effects of dilaton gravity are reflected in two ways. First, as the increasing of the parameter $\alpha, T_{\max }$ and $T_{\min }$ both increase. Secondly, the corresponding $\delta L$ also increases when $\alpha$ increases. This phenomenon is more apparent for large $\alpha$. Comparing Fig. 1 with 2, one may find that the case $n=4$ has higher $T_{\max }$ and $T_{\min }$ than the case $n=3$. This can also be witnessed by comparing Fig. 3 with 4 . On the other hand, the effect of the boundary region size is quite apparent in the range of the $\delta L$ axis when one compares Fig. 1 with 3 or compares Fig. 2 with 4.

\section{Numerical check of equal area law in $T-\delta L$ graph}

According to the analogous specific heat for $T-\delta L$ graph introduced in Ref. [4]

$C=T \frac{\partial \delta L}{\partial T}$

The $T-\delta L$ graph for $q<q_{c}$ can be divided into three branches. Namely the stable large radius branch with pos- 
Fig. $5 F$ vs. $T$ for

$n=3, l=1, b=1, k=1$.

a $\alpha=0, q=0.12$,

b $\alpha=0.25, q=0.12$,

c $\alpha=0.50, q=0.06$,

d $\alpha=0.75, q=0.06$

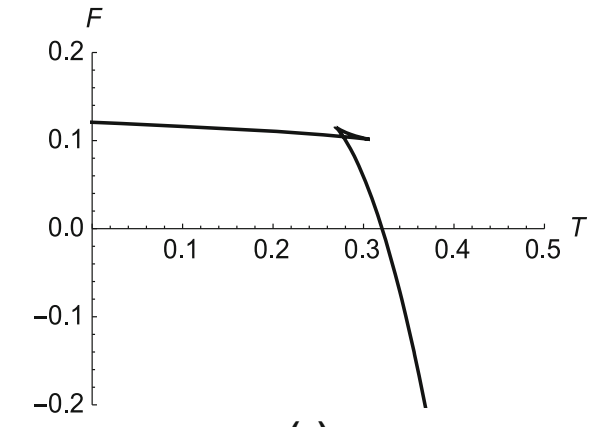

(a)

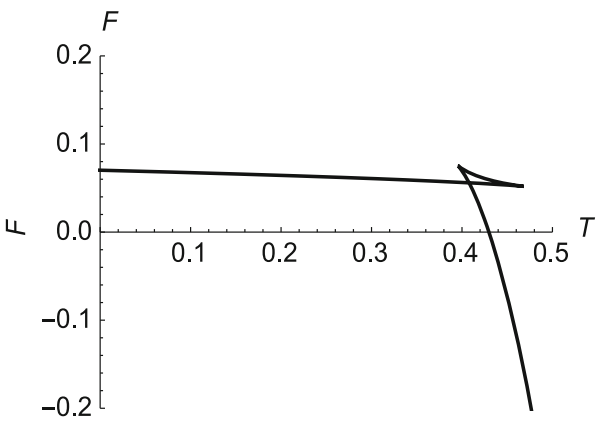

(c)

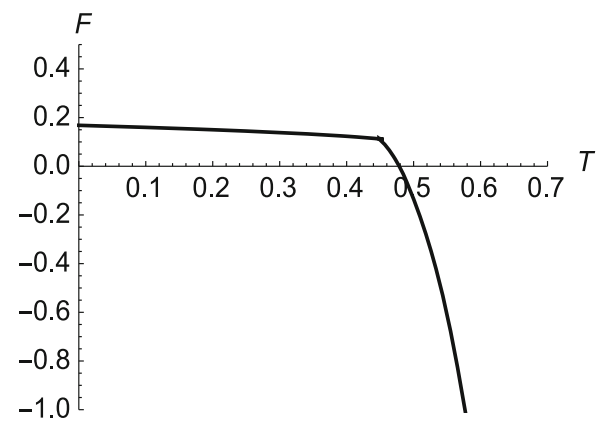

(a)

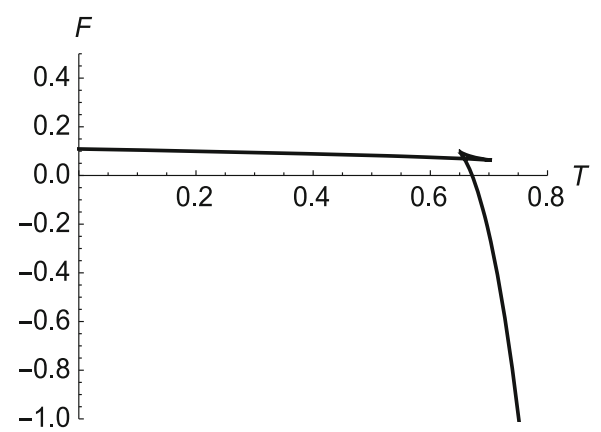

(c)

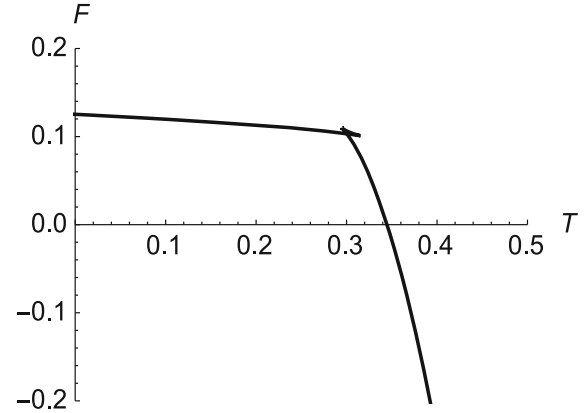

(b)

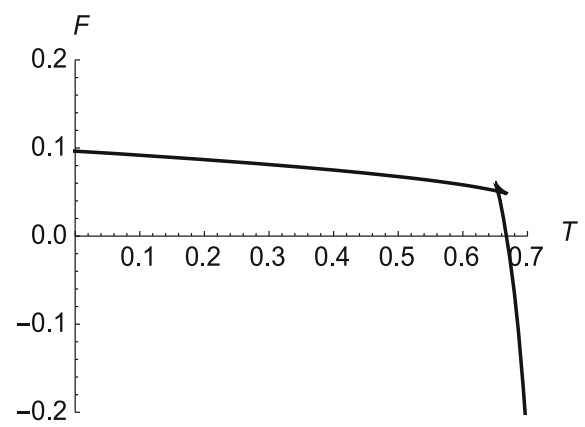

(d)
Fig. $6 F$ vs. $T$ for $n=4, l=1, b=1, k=1$.

a $\alpha=0, q=0.12$,

b $\alpha=0.25, q=0.12$,

c $\alpha=0.50, q=0.06$,

d $\alpha=0.75, q=0.06$

Table 1 Numerical check of equal area law in $T-\delta L$ graph for $n=3, \theta_{0}=0.2, l=1, b=1, k=1$

\begin{tabular}{llllllll}
\hline$\alpha$ & $q$ & $T_{*}$ & $\delta L_{1}$ & $\delta L_{3}$ & $T_{*}\left(\delta L_{3}-\delta L_{1}\right)$ & $\int_{\delta L_{1}}^{\delta L_{3}} T d \delta L$ & Relative error $(\%)$ \\
\hline 0 & 0.12 & 0.277496 & 0.0000637680 & 0.0002613560 & 0.0000548299 & 0.0000549115 & 0.1486 \\
0.25 & 0.12 & 0.3002889 & 0.0007501943 & 0.0030534139 & 0.0006916313 & 0.0006854523 & 0.9014 \\
0.50 & 0.06 & 0.4078042 & 0.0014369702 & 0.0157774345 & 0.0058481016 & 0.0058594007 & 0.1928 \\
0.75 & 0.06 & 0.65401625 & 0.0130252955 & 0.1416948822 & 0.0841520006 & 0.0841644278 & 0.0148 \\
\hline
\end{tabular}


Table 2 Numerical check of equal area law in $T-\delta L$ graph for $n=4, \theta_{0}=0.2, l=1, b=1, k=1$

\begin{tabular}{llllllll}
\hline$\alpha$ & $q$ & $T_{*}$ & $\delta L_{1}$ & $\delta L_{3}$ & $T_{*}\left(\delta L_{3}-\delta L_{1}\right)$ & $\int_{\delta L_{1}}^{\delta L_{3}} T d \delta L$ & Relative error $(\%)$ \\
\hline 0 & 0.12 & 0.448178 & 0.0000688919 & 0.0003011692 & 0.0001041016 & 0.0001041083 & 0.0064 \\
0.25 & 0.12 & 0.490898 & 0.0001308121 & 0.0005050468 & 0.0001837111 & 0.0001837165 & 0.0029 \\
0.50 & 0.06 & 0.65793667 & 0.0002371822 & 0.0046525001 & 0.0029049996 & 0.0029063836 & 0.0476 \\
0.75 & 0.06 & 1.070886315 & 0.0027446929 & 0.1854763902 & 0.1956848740 & 0.1957787303 & 0.0479 \\
\hline
\end{tabular}

Table 3 Numerical check of equal area law in $T-\delta L$ graph for $n=3, \theta_{0}=0.3, l=1, b=1, k=1$

\begin{tabular}{llllllll}
\hline$\alpha$ & $q$ & $T_{*}$ & $\delta L_{1}$ & $\delta L_{3}$ & $T_{*}\left(\delta L_{3}-\delta L_{1}\right)$ & $\int_{\delta L_{1}}^{\delta L_{3}} T d \delta L$ & Relative error $(\%)$ \\
\hline 0 & 0.12 & 0.277496 & 0.0017837032 & 0.0073122615 & 0.0015341528 & 0.0015366458 & 0.1622 \\
0.25 & 0.12 & 0.3002889 & 0.0024892165 & 0.0101203310 & 0.0022915390 & 0.0022710854 & 0.9006 \\
0.50 & 0.06 & 0.4078042 & 0.0042562477 & 0.0460392623 & 0.0170392888 & 0.0170769726 & 0.2207 \\
0.75 & 0.06 & 0.65401625 & 0.0301340104 & 0.3145252201 & 0.1859964725 & 0.1860497056 & 0.0286 \\
\hline
\end{tabular}

Table 4 Numerical check of equal area law in $T-\delta L$ graph for $n=4, \theta_{0}=0.3, l=1, b=1, k=1$

\begin{tabular}{llllllll}
\hline$\alpha$ & $q$ & $T_{*}$ & $\delta L_{1}$ & $\delta L_{3}$ & $T_{*}\left(\delta L_{3}-\delta L_{1}\right)$ & $\int_{\delta L_{1}}^{\delta L_{3}} T d \delta L$ & Relative error $(\%)$ \\
\hline 0 & 0.12 & 0.448178 & 0.0003666396 & 0.0015882967 & 0.0005475198 & 0.0005475520 & 0.0059 \\
0.25 & 0.12 & 0.490898 & 0.0006709414 & 0.0025817161 & 0.0009379955 & 0.0009380156 & 0.0021 \\
0.50 & 0.06 & 0.65793667 & 0.0011076791 & 0.0213463021 & 0.0133157322 & 0.0133239547 & 0.0617 \\
0.75 & 0.06 & 1.070886315 & 0.0104051412 & 0.5641278672 & 0.5929740896 & 0.5938277878 & 0.1438 \\
\hline
\end{tabular}

itive specific heat, the unstable medium radius branch with negative specific heat and the stable small radius branch with positive specific heat. This phenomenon is quite similar to that of the $T-S$ graph and the $P-v$ graph of dilaton black holes.

The unstable branch in the $T-\delta L$ curve with a bar $T=T_{*}$ vertical to the temperature axis can be removed by mimicking the approach of the $T-S$ graph [3]. Note that $T_{*}$ should be interpreted physically as the first order phase transition temperature and can be determined utilizing the free energy analysis. The analogous equal area law for the $T-\delta L$ graph reads

$T_{*} \times\left(\delta L_{3}-\delta L_{1}\right)=\int_{\delta L_{1}}^{\delta L_{3}} T d \delta L$,

where $\delta L_{1}, \delta L_{2}, \delta L_{3}$ are three values of $\delta L$ corresponding to $T_{*}$. Here we have assumed $\delta L_{1}<\delta L_{2}<\delta L_{3}$. If it can be proven that the LHS of Eq. (15) equals its RHS the conclusion can be drawn that the analogous equal area law holds for the $T-\delta L$ graph of dilaton black holes.

Before we carry out the examination, it is urgent to study the behavior of the free energy first. Utilizing Eqs. (4), (6), (9) and (10), the free energy of dilaton black holes can be derived,

$$
\begin{aligned}
F= & M-T S \\
= & \frac{\omega_{n-1}\left(1+\alpha^{2}\right) b^{(n-1) \gamma} r_{+}^{n-2+\gamma-n \gamma}}{16 \pi} \\
& \times\left\{\frac{k(n-2) r_{+}^{2 \gamma} b^{-2 \gamma}}{n-2+\alpha^{2}}+\frac{n b^{2 \gamma} r_{+}^{2-2 \gamma}\left(\alpha^{2}-1\right)}{l^{2}\left(n-\alpha^{2}\right)}\right. \\
& \left.+\frac{2 b^{-2(n-2) \gamma} q^{2} r_{+}^{2(n-2)(\gamma-1)}\left(2 n-3+\alpha^{2}\right)}{(n-1)\left(n-2+\alpha^{2}\right)}\right\},
\end{aligned}
$$

with the $F-T$ graphs depicted in Figs. 5 and 6 for different choices of parameters.

The familiar swallow tail behavior can be observed in all the $F-T$ graphs and $T_{*}$ can be obtained from the intersection point of two branches in the graphs. Comparing these graphs, one may conclude that the first order phase transition temperature $T_{*}$ is affected by both $\alpha$ and $n$. When $\alpha$ increases, $T_{*}$ increases. When $n$ increases, $T_{*}$ increases too. With $T_{*}$ at hand the left-hand side and right-hand side of Eq. (15) can be calculated for different cases. As can be seen from Tables 1, 2, 3 and 4, the relative errors for all the cases are small enough and we can safely conclude that the analogous equal area law holds for the $T-\delta L$ graph of dilaton black holes. 


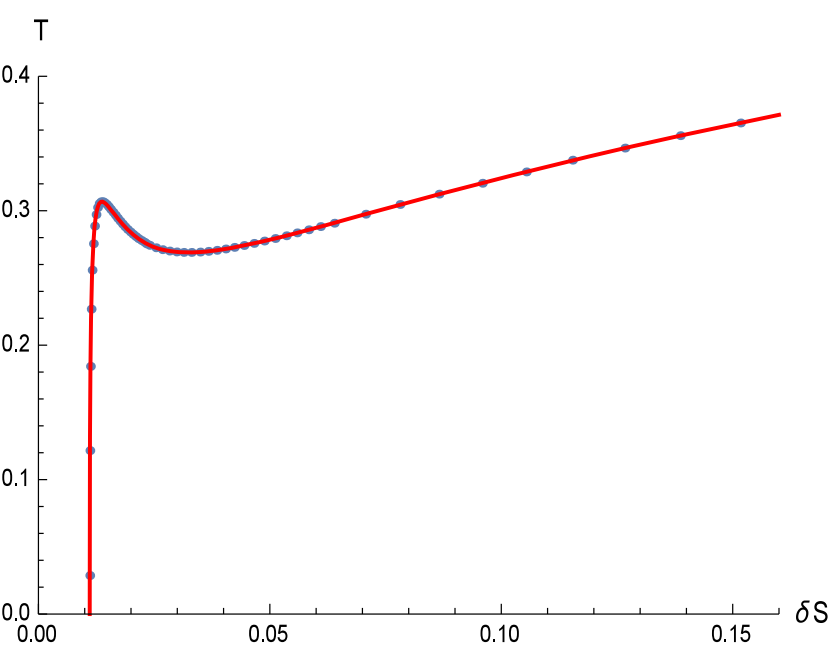

(a)

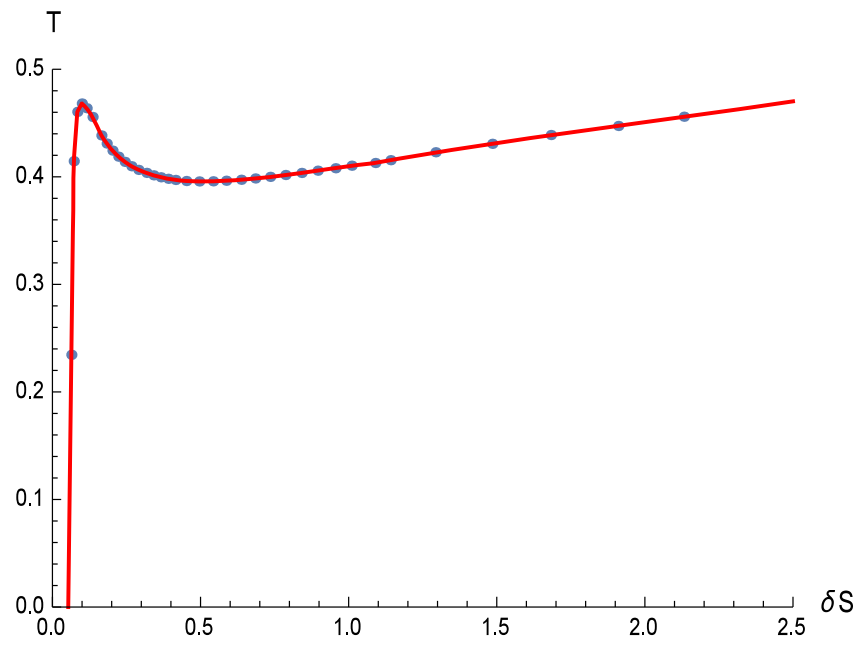

(c)

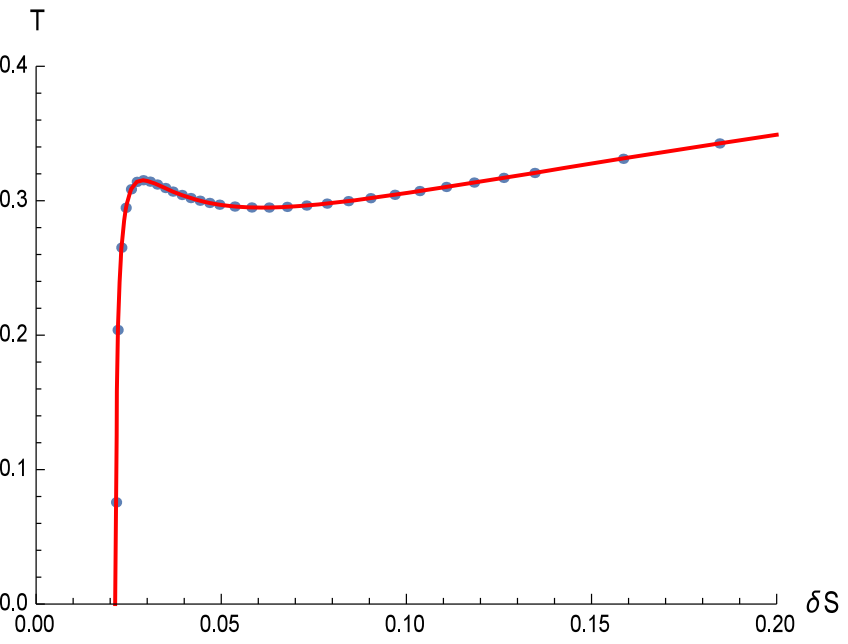

(b)

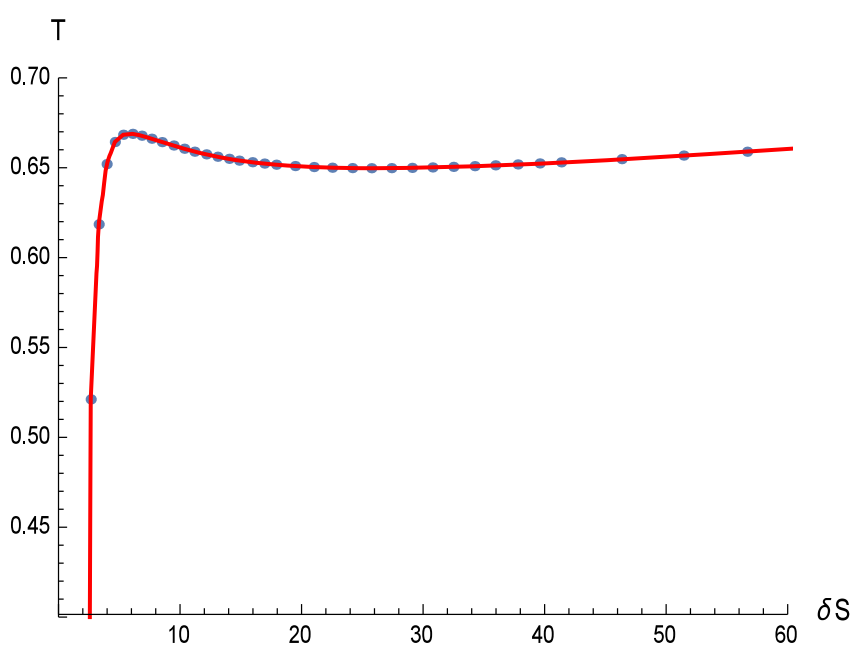

(d)

Fig. $7 T$ vs. $\delta S$ for $n=3, \theta_{0}=0.2$. $\mathbf{a} \alpha=0, q=0.12, \mathbf{b} \alpha=0.25, q=0.12, \mathbf{c} \alpha=0.5, q=0.06, \mathbf{d} \alpha=0.75, q=0.06$

\section{Entanglement entropy of dilaton black holes and its van der Waals like behavior}

Beside the two point correlation function, entanglement entropy serves as another nonlocal observable to probe the holographic properties of black holes.

The entanglement entropy can be expressed holographically in terms of the area of a minimal surface anchored on $\partial A$ as $[51,52]$

$S_{A}=\frac{\operatorname{Area}(\Sigma)}{4 G_{N}}$,

where $\Sigma$ is the codimension- 2 minimal surface with boundary condition $\partial \Sigma=\partial A$ and $G_{N}$ is the Newton constant.

As argued in Ref. [2], dealing with the phase transition between connected and disconnected minimal surfaces can be avoided by avoiding large entangling regions. Taking this into consideration, we choose the region to be a spherical cap on the boundary delimited by $\theta \leq \theta_{0}$.

Parametrizing the minimal surface by the function $r(\theta)$, the holographic entanglement entropy can be obtained:

$S_{A}=\frac{\pi}{2} \int_{0}^{\theta_{0}} r \sin \theta \sqrt{\frac{r^{\prime 2}}{f(r)}+r^{2}} \mathrm{~d} \theta$,

where $r^{\prime}=\mathrm{d} r / \mathrm{d} \theta$. From the above equation, the following can be obtained:

$\mathcal{L}=r \sin \theta \sqrt{\frac{r^{\prime 2}}{f(r)}+r^{2}}$.

Substituting it into an Euler-Lagrange equation, the equation for $r(\theta)$ can be derived. Then $r(\theta)$ can be obtained by solving the equation constrained by the boundary condition $r(0)=$ $r_{0}, r^{\prime}(0)=0$ numerically. 


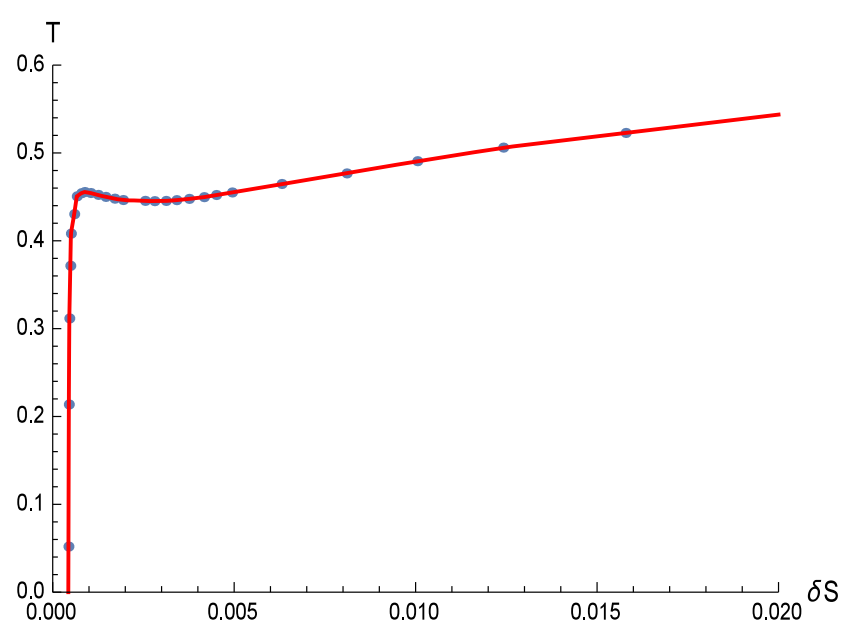

(a)

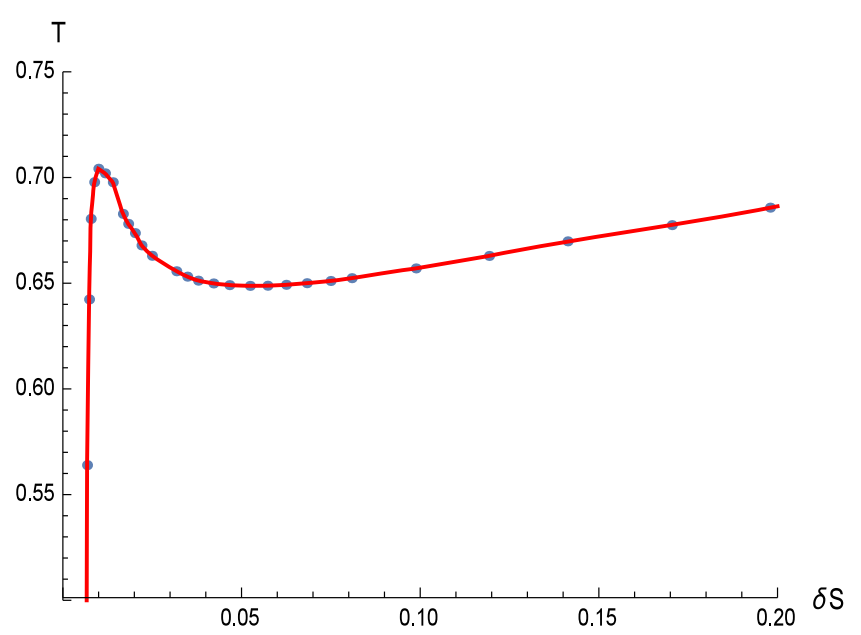

(c)

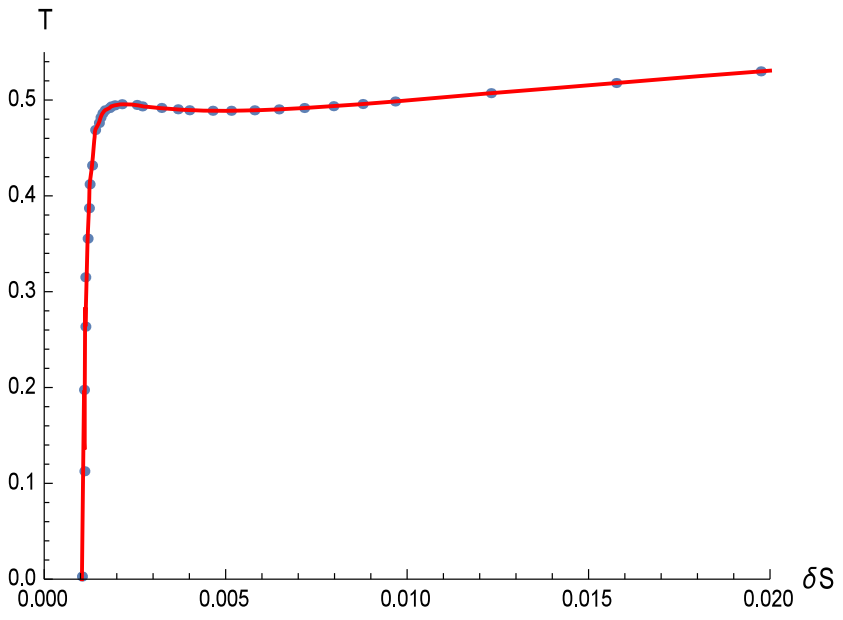

(b)

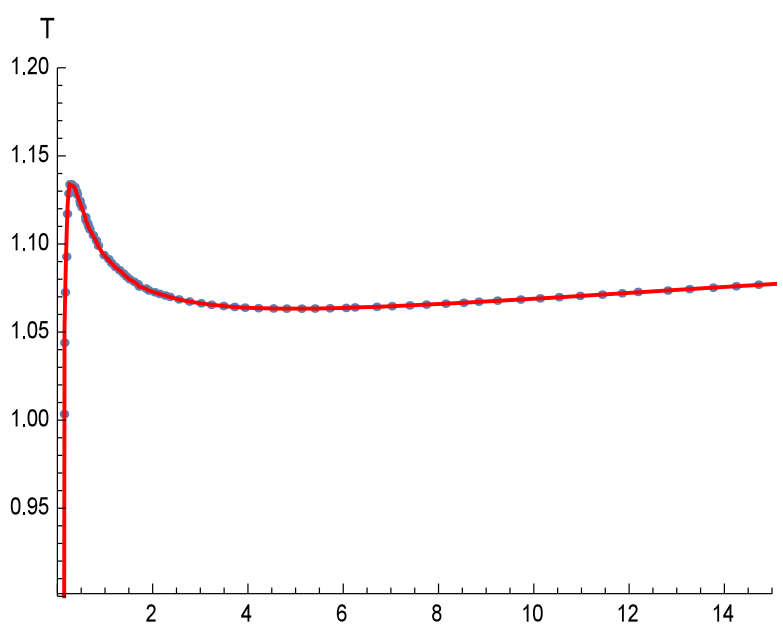

(d)

Fig. $8 T$ vs. $\delta S$ for $n=4, \theta_{0}=0.2$. a $\alpha=0, q=0.12, \mathbf{b} \alpha=0.25, q=0.12, \mathbf{c} \alpha=0.5, q=0.06, \mathbf{d} \alpha=0.75, q=0.06$

To avoid the divergence the holographic entanglement entropy should be regularized by subtracting the entanglement entropy $S_{0}$ ( $S_{0}$ can also be obtained through numerical treatment) in pure AdS with the same boundary region. We denote the regularized entanglement entropy as $\delta S$ and in the following discussions mainly deal with the behavior of the $T-\delta S$ graph.

As in Sect. 3 we will consider $4 \times 2 \times 2=16$ cases due to different choices of parameters. For the sake of clarity, we repeat the choices here. $\alpha$ is respectively chosen as $0,0.25,0.5,0.75$ in order to investigate the effect of dilaton gravity on the phase structure of holographic entanglement entropy while $n$ respectively is chosen as 3, 4 in order to probe the effect of spacetime dimensionality. $\theta_{0}$, respectively, is chosen as $0.2,0.3$ in order to study the effect of different boundary region sizes. Accordingly, the cutoff $\theta_{c}$ will also be chosen as $0.199,0.299$. In each case we focus on the case $q<q_{c}$ to probe the possible van der Waals behavior.

We plot the cases in which $n=3, \theta_{0}=0.2$ in Fig. 7a-d and the cases in which $n=4, \theta_{0}=0.2$ in Fig. 8a, b. The cases in which $n=3, \theta_{0}=0.3$ are displayed in Fig. 9a, b while the cases in which $n=4, \theta_{0}=0.3$ are depicted in Fig. 10a-d.

The van der Waals like behavior can be clearly witnessed from all the $T-\delta S$ graphs for $q<q_{c}$. As seen in the $T-$ $\delta L$ graphs presented in Sect. 3, there also exists both a local maximum temperature and a local minimum temperature in all the $T-\delta S$ graphs. As depicted in Figs. 7, 8, 9 and 10, not only $T_{\max }$ and $T_{\min }$ but also the corresponding $\delta S$ increases as the parameter $\alpha$ is increasing, showing the impact of dilaton gravity. Comparing Fig. 7 with 8, or comparing Fig. 9 with 10 , it is not difficult to observe that the cases $n=4$ have higher $T_{\max }$ and $T_{\min }$ than the cases $n=3$. Moreover, the 


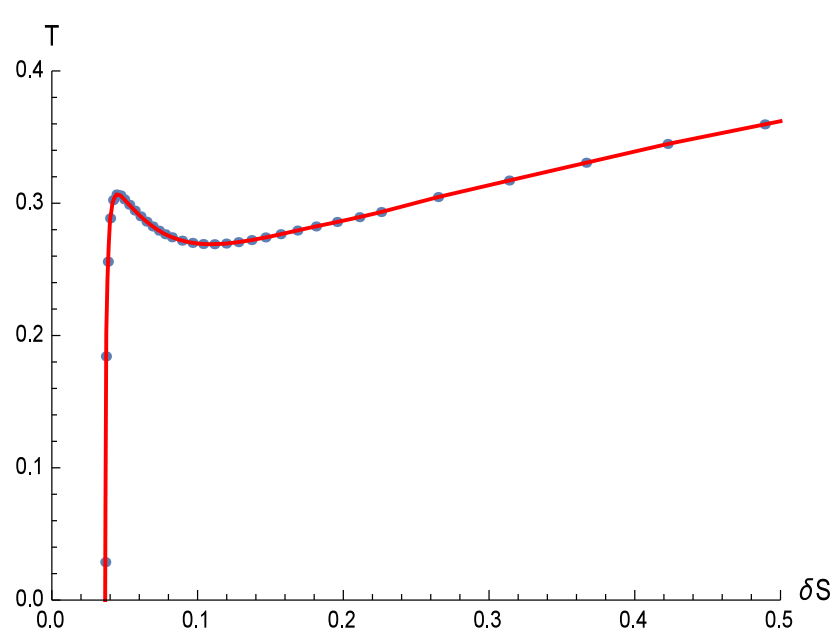

(a)

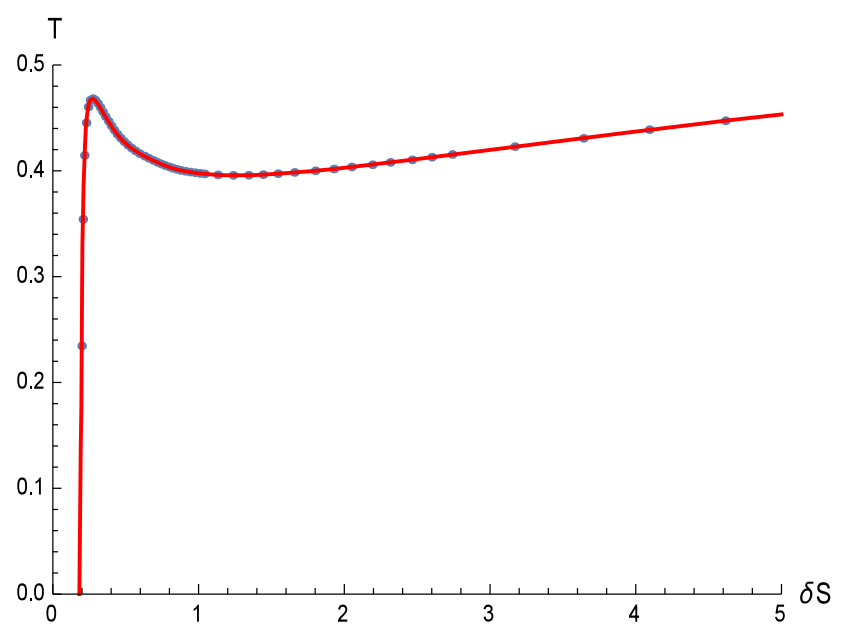

(c)

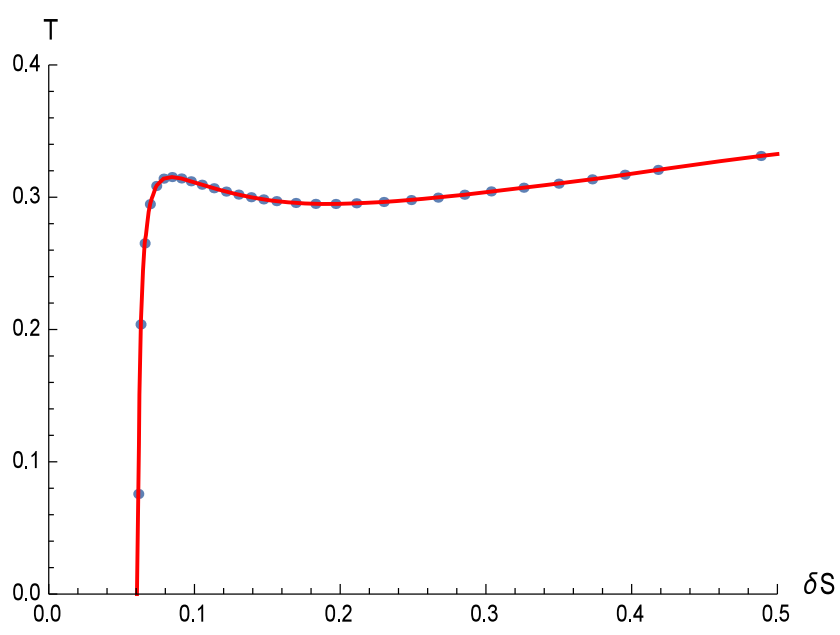

(b)

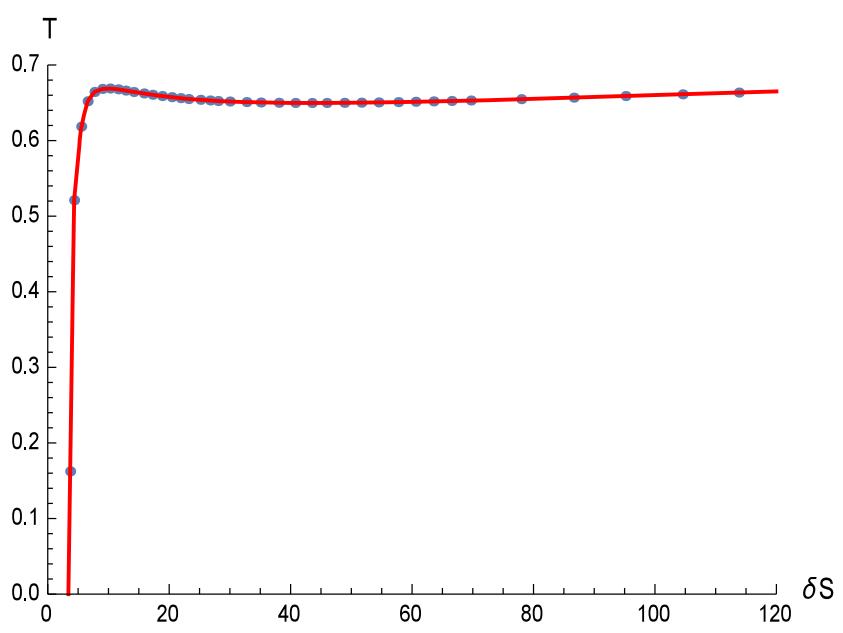

(d)

Fig. $9 T$ vs. $\delta S$ for $n=3, \theta_{0}=0.3$. $\mathbf{a} \alpha=0, q=0.12, \mathbf{b} \alpha=0.25, q=0.12, \mathbf{c} \alpha=0.5, q=0.06, \mathbf{d} \alpha=0.75, q=0.06$

range of the $\delta S$ axis for the cases $\theta_{0}=0.2$ differs from that of the cases $\theta_{0}=0.3$, showing the effect of the boundary region size. These phenomena are quite similar to those of two point correlation function probed in Sect. 3 .

\section{Numerical check of equal area law in $T-\delta S$ graph}

The analogous specific heat for $T-\delta S$ graph has been defined in the literature [4] as

$C=T \frac{\partial \delta S}{\partial T}$.

Based on the above definition each $T-\delta S$ graph for $q<$ $q_{c}$ can be divided into three branches. Namely, the stable large radius branch with positive specific heat, the unstable medium radius branch with negative specific heat and the stable small radius branch with positive specific heat. This phenomenon is quite similar to that of the $T-S$ graph and $P-v$ graph of dilaton black holes. It is also similar to that of the $T-\delta L$ graph discussed in Sect. 3.

Taking a similar treatment the unstable branch in the $T$ $\delta S$ curve with a bar $T=T_{*}$ vertical to the temperature axis can be removed. The analogous equal area law for the $T-\delta S$ graph can be written

$T_{*} \times\left(\delta S_{3}-\delta S_{1}\right)=\int_{\delta S_{1}}^{\delta S_{3}} T d \delta S$,

where $\delta S_{1}, \delta S_{2}, \delta S_{3}$ are three values of $\delta S$ corresponding to $T_{*}$ with the assumption that $\delta S_{1}<\delta S_{2}<\delta S_{3}$.

The left-hand side and right-hand side of Eq. (21) are calculated for different cases and the results are listed in Tables $5,6,7$ and 8 , from which it is clearly seen that the relative errors for all the cases are amazingly small. So we can safely draw the conclusion that the analogous equal area law Eq. (21) holds for $T-\delta S$ graph of dilaton black holes. 


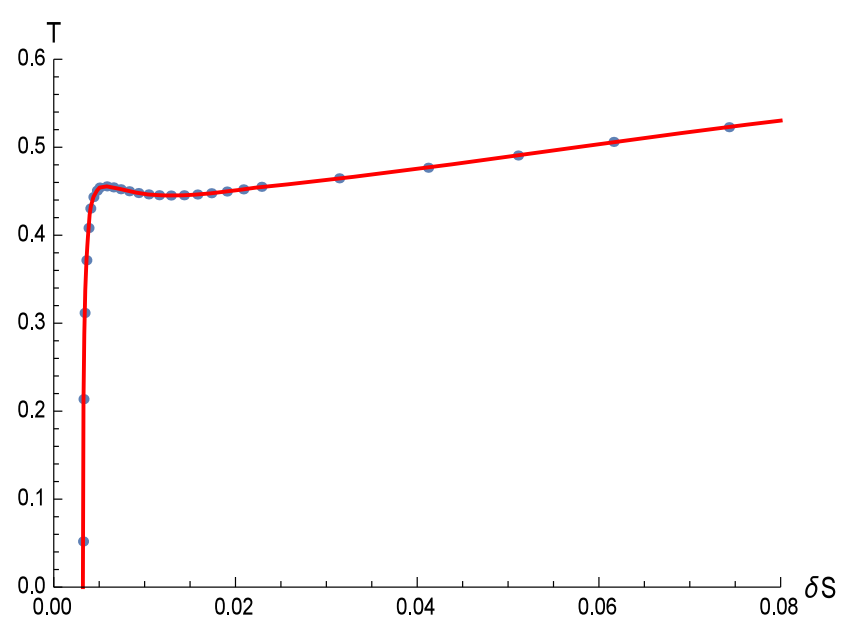

(a)

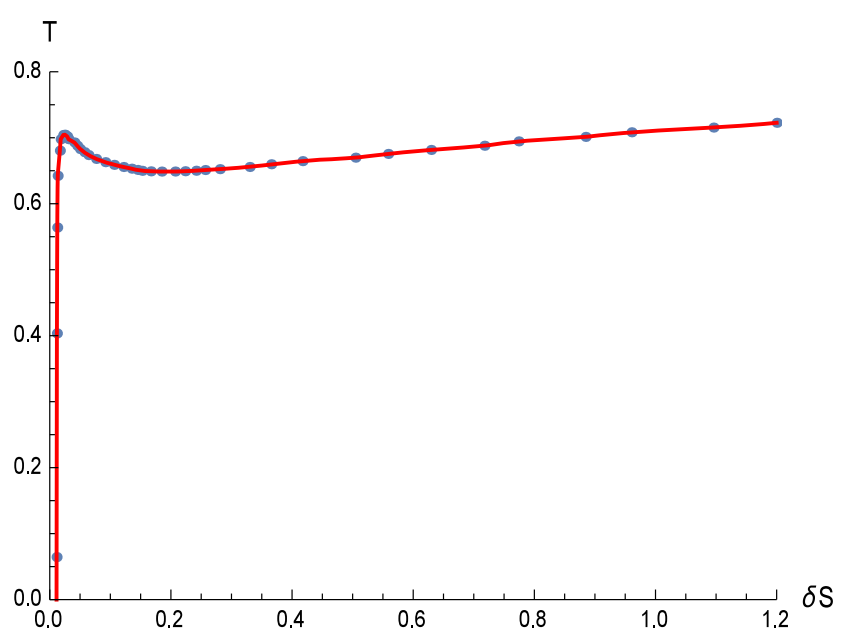

(c)

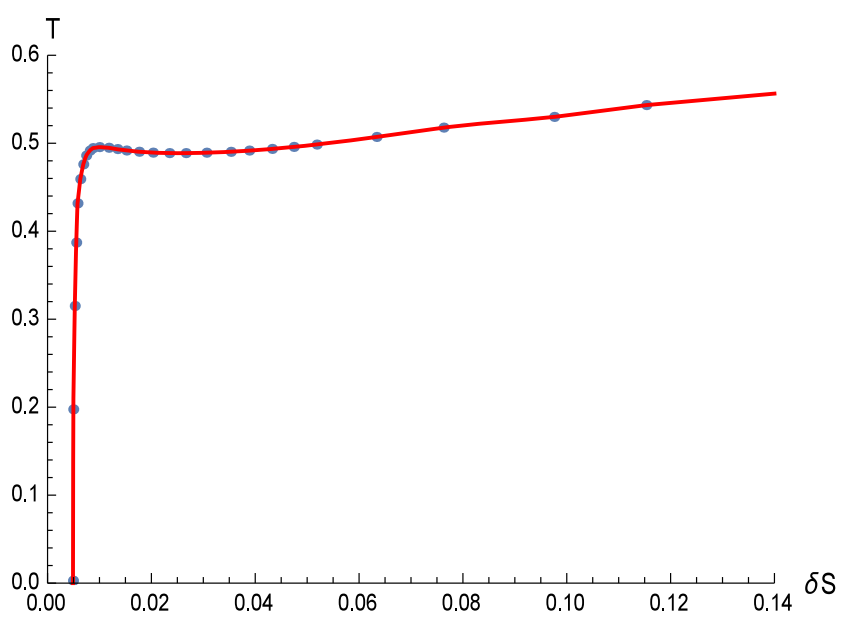

(b)

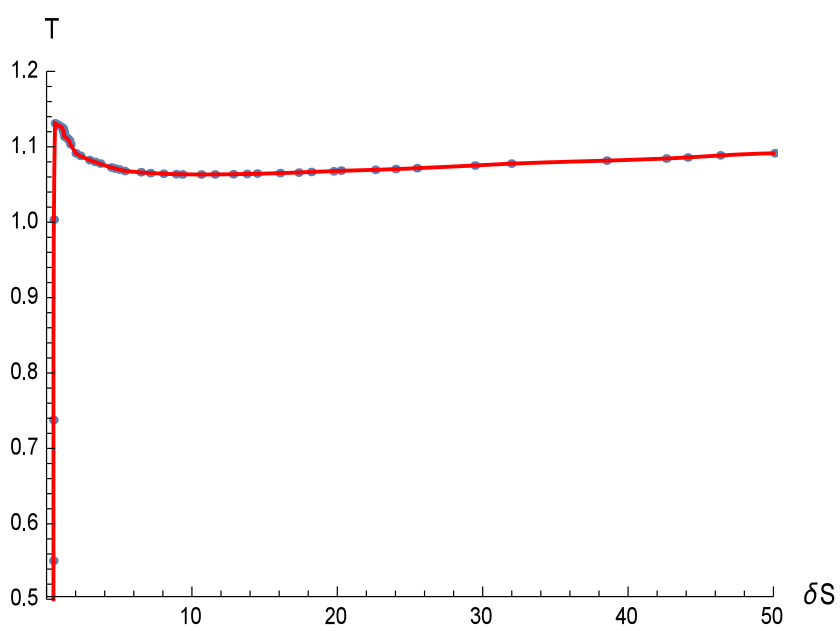

(d)

Fig. $10 T$ vs. $\delta S$ for $n=4, \theta_{0}=0.3$. a $\alpha=0, q=0.12$, $\mathbf{b} \alpha=0.25, q=0.12, \mathbf{c} \alpha=0.5, q=0.06, \mathbf{d} \alpha=0.75, q=0.06$

Table 5 Numerical check of equal area law in $T-\delta S$ graph for $n=3, \theta_{0}=0.2, l=1, b=1, k=1$

\begin{tabular}{lllllrrr}
\hline$\alpha$ & $q$ & $T_{*}$ & \multicolumn{1}{l}{$\delta S_{1}$} & \multicolumn{1}{c}{$\delta S_{3}$} & $T_{*}\left(\delta S_{3}-\delta S_{1}\right)$ & $\int_{\delta S_{1}}^{\delta S_{3}} T d \delta S$ & Relative error $(\%)$ \\
\hline 0 & 0.12 & 0.277496 & 0.0119147123 & 0.0488420932 & 0.0102472005 & 0.0102600904 & 0.1256 \\
0.25 & 0.12 & 0.3002889 & 0.0247660099 & 0.0858928910 & 0.0183557239 & 0.0183641401 & 0.0458 \\
0.50 & 0.06 & 0.4078042 & 0.0716592283 & 0.9511787777 & 0.3586717662 & 0.3592798682 & 0.1693 \\
0.75 & 0.06 & 0.65401625 & 4.0720587420 & 44.1845284093 & 26.2342069900 & 26.2343780610 & 0.0007 \\
\hline
\end{tabular}

Table 6 Numerical check of equal area law in $T-\delta S$ graph for $n=4, \theta_{0}=0.2, l=1, b=1, k=1$

\begin{tabular}{llllrrrr}
\hline$\alpha$ & $q$ & $T_{*}$ & $\delta S_{1}$ & \multicolumn{1}{l}{$\delta S_{3}$} & $T_{*}\left(\delta S_{3}-\delta S_{1}\right)$ & $\int_{\delta S_{1}}^{\delta S_{3}} T d \delta S$ & Relative error $(\%)$ \\
\hline 0 & 0.12 & 0.448178 & 0.0006548237 & 0.0038527782 & 0.0014332529 & 0.0014330141 & 0.0167 \\
0.25 & 0.12 & 0.490898 & 0.0017571468 & 0.0067818433 & 0.0024666135 & 0.0024663505 & 0.0107 \\
0.50 & 0.06 & 0.65793667 & 0.0075207833 & 0.1020304981 & 0.0621814070 & 0.0622185738 & 0.0597 \\
0.75 & 0.06 & 1.070886315 & 0.1595664149 & 11.1997305875 & 11.8227607278 & 11.8253260328 & 0.0217 \\
\hline
\end{tabular}


Table 7 Numerical check of equal area law in $T-\delta S$ graph for $n=3, \theta_{0}=0.3, l=1, b=1, k=1$

\begin{tabular}{lllllrrr}
\hline$\alpha$ & $q$ & $T_{*}$ & \multicolumn{1}{l}{$\delta S_{1}$} & \multicolumn{1}{c}{$\delta S_{3}$} & $T_{*}\left(\delta S_{3}-\delta S_{1}\right)$ & $\int_{\delta S_{1}}^{\delta S_{3}} T d \delta S$ & \multicolumn{1}{c}{ Relative error $(\%)$} \\
\hline 0 & 0.12 & 0.277496 & 0.0394539472 & 0.1608172794 & 0.0336778392 & 0.0337657232 & 0.2603 \\
0.25 & 0.12 & 0.3002889 & 0.0711468332 & 0.2719042091 & 0.0602852116 & 0.0603586328 & 0.1216 \\
0.50 & 0.06 & 0.4078042 & 0.2149538454 & 2.3059365730 & 0.8527115384 & 0.8534806310 & 0.0901 \\
0.75 & 0.06 & 0.65401625 & 6.7549216837 & 74.3949477659 & 44.2376762082 & 44.2373479097 & 0.0007 \\
\hline
\end{tabular}

Table 8 Numerical check of equal area law in $T-\delta S$ graph for $n=4, \theta_{0}=0.3, l=1, b=1, k=1$

\begin{tabular}{llllrrrr}
\hline$\alpha$ & $q$ & $T_{*}$ & $\delta S_{1}$ & \multicolumn{1}{c}{$\delta S_{3}$} & $T_{*}\left(\delta S_{3}-\delta S_{1}\right)$ & $\int_{\delta S_{1}}^{\delta S_{3}} T d \delta S$ & \multicolumn{1}{c}{ Relative error $(\%)$} \\
\hline 0 & 0.12 & 0.448178 & 0.0046156698 & 0.0177350761 & 0.0058798293 & 0.0058821069 & 0.0387 \\
0.25 & 0.12 & 0.490898 & 0.0080413189 & 0.0369024703 & 0.0141678815 & 0.0141621430 & 0.0405 \\
0.50 & 0.06 & 0.65793667 & 0.0145412501 & 0.3493654246 & 0.2202931024 & 0.2207464643 & 0.2054 \\
0.75 & 0.06 & 1.070886315 & 0.5398143877 & 24.4969431344 & 25.6553613215 & 25.6488620105 & 0.0253 \\
\hline
\end{tabular}

\section{Conclusions}

In this paper we probe the critical phenomena of dilaton black holes from a perspective totally different from the $P-v$ criticality and the $q-U$ criticality discussed in the literature [35].

On the one hand we study the two point correlation function of dilaton black holes. Considering the points $\left(t_{0}, x_{i}\right)$ and $\left(t_{0}, x_{j}\right)$ on the boundary the equal time two point correlation function can be written in the large $\Delta$ limit and the proper length can be obtained by parameterizing the trajectory with $\theta$. Applying the Euler-Lagrange equation the equation of motion can be derived for $r(\theta)$. Solving this equation constrained by the boundary condition $r(0)=r_{0}, r^{\prime}(0)=0$, we obtain $r(\theta)$ via numerical methods. To avoid the divergence, we regularize the geodesic length by subtracting the geodesic length $L_{0}$ in the pure AdS case with the same boundary region.

On the other hand, we investigate the entanglement entropy of dilaton black holes which can be expressed holographically in terms of the area of a minimal surface $\Sigma$ anchored on $\partial A$ with boundary condition $\partial \Sigma=\partial A$. We choose the region to be a spherical cap on the boundary delimited by $\theta \leq \theta_{0}$ and obtain the holographic entanglement entropy through parametrizing the minimal surface by $r(\theta)$, utilizing the Euler-Lagrange equation again, we derive the equation for $r(\theta)$. Solving the equation constrained by the boundary condition numerically, we obtain $r(\theta)$. For a similar consideration, we regularize the entanglement entropy by subtracting the entanglement entropy $S_{0}$ in pure AdS with the same boundary region.

For both the two point correlation function and the entanglement entropy, we consider $4 \times 2 \times 2=16$ cases due to different choices of parameters. Namely, $\alpha$, respectively, is chosen as $0,0.25,0.5,0.75$ in order to probe the effect of dilaton gravity on the phase structure, while $n$ respec- tively is chosen as 3, 4 in order to study the impact of spacetime dimensionality. $\theta_{0}$, respectively, is chosen as $0.2,0.3$ in order to check the effect of different boundary region sizes. The behavior of the $T-\delta L$ graphs is quite similar to that of the $T-\delta S$ graphs. First, the van der Waals like behavior can be clearly witnessed from all the $T-\delta L(T-\delta S)$ graphs for $q<q_{c}$. Second, there exist both the local maximum temperature and the local minimum temperature. Third, not only $T_{\max }$ and $T_{\min }$ but also the corresponding $\delta L(\delta S)$ increase as the parameter $\alpha$ is increasing, showing the impact of dilaton gravity. This phenomenon is more apparent for large $\alpha$. Fourth, $T_{\max }$ and $T_{\min }$ of the cases $n=4$ is higher than that of the cases $n=3$. Finally, the effect of the boundary region size is quite apparent in the range of $\delta L(\delta S)$ axis.

Furthermore, we discuss the stability of dilaton black holes by introducing the analogous specific heat for $T-\delta L$ graph and $T-\delta S$ graph accordingly. All these graphs can be divided into three branches. Namely, the stable large radius branch with positive specific heat, the unstable medium radius branch with negative specific heat and the stable small radius branch with positive specific heat. This phenomenon is quite similar to that of the $T-S$ graph and the $P-v$ graph of dilaton black holes in the former research. To remove the unstable branch, we introduce a bar $T=T_{*}$ vertical to the temperature axis. $T_{*}$ should be interpreted physically as the first order phase transition temperature and can be determined utilizing the free energy analysis. It is shown that the first order phase transition temperature $T_{*}$ is affected by both $\alpha$ and $n$. When $\alpha$ increases, $T_{*}$ increases. When $n$ increases, $T_{*}$ increases too. We also examine the analogous equal area law for both the $T-\delta L$ graph and the $T-\delta S$ graph. The relative errors for all the cases are small enough that we can safely conclude that the analogous equal area law holds for the $T-\delta L(T-\delta S)$ graph of dilaton black holes. 
Acknowledgements The author would like to express his sincere gratitude to Dr. Jieci Wang, Dr. Xiongjun Fang, Dr. Zixu Zhao, Prof. Jia-Lin Zhang, Prof. Qiyuan Pan, Prof. Songbai Chen and Prof. Jiliang Jing for their warm hospitality during the Summer School and Workshop on Gravitation and Cosmology held in Hunan Normal University. The author also want to thank Dr. Xiao-Xiong Zeng for helpful discussions on numerics. The author is supported by National Natural Science Foundation of China (Grant No. 11605082) and Guangdong Natural Science Foundation of China (Grant No. 2016A030310363). He is also partly supported by Guangdong Natural Science Foundation of China (Grant Nos. 2016A030307051, 2015A030313789).

Open Access This article is distributed under the terms of the Creative Commons Attribution 4.0 International License (http://creativecomm ons.org/licenses/by/4.0/), which permits unrestricted use, distribution, and reproduction in any medium, provided you give appropriate credit to the original author(s) and the source, provide a link to the Creative Commons license, and indicate if changes were made.

Funded by SCOAP . $^{3}$

\section{References}

1. C.V. Johnson, Large $N$ phase transitions, finite volume, and entanglement entropy. JHEP 1403, 047 (2014)

2. P.H. Nguyen, An equal area law for holographic entanglement entropy of the AdS-RN black hole. JHEP 1512, 139 (2015)

3. E. Spallucci, A. Smailagic, Maxwell's equal area law for charged Anti-de Sitter black holes. Phys. Lett. B 723, 436-441 (2013)

4. X.X. Zeng, X.M. Liu, L.F. Li, Phase structure of the BornInfeld-anti-de Sitter black holes probed by non-local observables. arXiv: 1601.01160

5. E. Caceres, P.H. Nguyen, J.F. Pedraza, Holographic entanglement entropy and the extended phase structure of STU black holes. JHEP 1509, $184(2015)$

6. X.X. Zeng, H. Zhang, L.F. Li, Phase transition of holographic entanglement entropy in massive gravity. Phys. Lett. B 756, 170179 (2016)

7. A. Dey, S. Mahapatra, T. Sarkar, Thermodynamics and entanglement entropy with Weyl corrections. arXiv: 1512.07117

8. X.X.Zeng, L.F. Li, Van der Waals phase transition in the framework of holography. arXiv: 1512.08855

9. S. Kundu, J.F. Pedraza, Aspects of holographic entanglement at finite temperature and chemical potential. arXiv:1602.07353

10. D. Momeni, K. Myrzakulov, R. Myrzakulov, Fidelity susceptibility as holographic $P-V$ criticality. arXiv: 1604.06909

11. J.X. Mo, G.Q. Li, Z.T. Lin, X.X. Zeng, Van der Waals like behavior and equal area law of two point correlation function of $f(R)$ AdS black holes. arXiv: 1604.08332

12. Y. Sun, H. Xu, L. Zhao, Thermodynamics and holographic entanglement entropy for spherical black holes in 5D Gauss-Bonnet gravity. arXiv: 1606.06531

13. G.W. Gibbons, K. Maeda, Black holes and membranes in higher dimensional theories with dilaton fields. Nucl. Phys. B 298, 741 (1988)

14. T. Koikawa, M. Yoshimura, Dilaton fields and event horizon. Phys. Lett. B 189, 29 (1987)

15. D. Garfinkle, G.T. Horowitz, A. Strominger, Charged black holes in string theory. Phys. Rev. D 43, 3140 (1991)

16. R. Gregory, J.A. Harvey, Black holes with a massive dilaton. Phys. Rev. D 47, 2411 (1993)

17. M. Rakhmanov, Dilaton black holes with electric charge. Phys. Rev. D 50, 5155 (1994)
18. K.C.K. Chan, J.H. Horne, R.B. Mann, Charged dilaton black holes with unusual asymptotics. Nucl. Phys. B 447, 441-464 (1995)

19. R.G. Cai, J.Y. Ji, K.S. Soh, Topological dilaton black holes. Phys. Rev D 57, 6547 (1998)

20. R.G. Cai, Y.Z. Zhang, Holography and brane cosmology in domain wall backgrounds. Phys. Rev. D 64, 104015 (2001)

21. G. Clement, D. Gal'tsov, C. Leygnac, Linear dilaton black holes. Phys. Rev. D 67, 024012 (2003)

22. G. Clement, C. Leygnac, Non-asymptotically flat, non-AdS dilaton black holes. Phys. Rev. D 70, 084018 (2004)

23. C.J. Gao, H.N. Zhang, Topological black holes in dilaton gravity theory. Phys. Lett. B 612, 127-136 (2006)

24. S.J. Poletti, D.L. Wiltshire, The global properties of static spherically symmetric charged dilaton space-times with a Liouville potential. Phys. Rev. D 50, 7260 (1994)

25. K. Goldstein, S. Kachru, S. Prakash, S.P. Trivedi, Holography of charged dilaton black holes. JHEP 1008, 078 (2010)

26. K. Goldstein, N. Lizuka, S. Kachru, S. Prakash, S.P. Trivedi, A. Westphal, Holography of dyonic dilaton black branes. JHEP 1010, 027 (2010)

27. A. Anabalon, D. Astefanesei, On attractor mechanism of $\mathrm{Ad} S_{4}$ black holes. Phys. Lett. B 727, 568-572 (2013)

28. D. Astefanesei, N. Banerjee, S. Dutta, Moduli and electromagnetic black brane holography. JHEP 1102, 021 (2011)

29. C.M. Chen, D.W. Peng, Holography of charged dilaton black holes in general dimensions. JHEP 1006, 093 (2010)

30. B. Gouteraux, B.S. Kim, R. Meyer, Charged dilatonic black holes and their transport properties. Fortschr. Phys. 59, 723 (2011)

31. S.S. Gubser, F.D. Rocha, Peculiar properties of a charged dilatonic black hole in $\operatorname{Ad} S_{5}$. Phys. Rev. D 81, 046001 (2010)

32. Y.C. Ong, P. Chen, Stringy stability of charged dilaton black holes with flat event horizon. JHEP 1208, 79 (2012)

33. W.J. Li, Some properties of the holographic fermions in an extremal charged dilatonic black holes. Phys. Rev. D 84, 064008 (2011)

34. W.J. Li, R. Meyer, H. Zhang, Holographic non-relativistic fermionic fixed point by the charged dilatonic black hole. JHEP 01, 153 (2012)

35. R. Zhao, H.H. Zhao, M.S. Ma, L.C. Zhang, On the critical phenomena and thermodynamics of charged topological dilaton AdS black holes. Eur. Phys. J. C 73, 2645 (2013)

36. M.H. Dehghani, S.H. Hendi, A. Sheykhi, H.R. Sedehi, Thermodynamics of rotating black branes in Einstein-Born-Infeld-dilaton gravity. JCAP 02, 020 (2007)

37. A. Sheykhi, N. Riazi, Thermodynamics of black holes in $(n+1)$ dimensional Einstein-Born-Infeld dilaton gravity. Phys. Rev. D 75, 024021 (2007)

38. S.H. Hendi, A. Sheykhi, M.H. Dehghani, Thermodynamics of higher dimensional topological charged AdS black branes in dilaton gravity. Eur. Phys. J. C 70, 703 (2010)

39. A. Sheykhi, M.H. Dehghani, S.H. Hendi, Thermodynamic instability of charged dilaton black holes in AdS spaces. Phys. Rev. D 81, 084040 (2010)

40. A. Sheykhi, S. Hajkhalili, Dilaton black holes coupled to nonlinear electrodynamic field. Phys. Rev. D 89, 104019 (2014)

41. A. Sheykhi, A. Kazemi, Higher dimensional dilaton black holes in the presence of exponential nonlinear electrodynamics. Phys. Rev. D 90, 044028 (2014)

42. M.H. Dehghani, S. Kamrani, A. Sheykhi, $P-V$ criticality of charged dilatonic black holes. Phys. Rev. D 90, 104020 (2014)

43. M.K. Zangeneh, A. Sheykhi, M.H. Dehghani, Thermodynamics of higher dimensional topological dilation black holes with a powerlaw Maxwell field. Phys. Rev. D 91, 044035 (2015)

44. M.K. Zangeneh, M.H. Dehghani, A. Sheykhi, Thermodynamics of charged rotating dilaton black branes with power-law Maxwell field. Eur. Phys. J. C 75, 497 (2015) 
45. A. Sheykhi, F. Naeimipour, S.M. Zebarjad, Phase transition and thermodynamic geometry of topological dilaton black holes in gravitating logarithmic nonlinear electrodynamics. Phys. Rev. D 91, 124057 (2015)

46. A. Sheykhi, F. Naeimipour, S.M. Zebarjad, Thermodynamic geometry and thermal stability of $n$-dimensional dilaton black holes in the presence of logarithmic nonlinear electrodynamics. Phys. Rev. D 92, 124054 (2015)

47. S.H. Hendi, A. Sheykhi, S. Panahiyan, B.E. Panah, Phase transition and thermodynamic geometry of Einstein-Maxwell-dilaton black holes. Phys. Rev. D 92, 064028(2015)
48. M.H. Dehghani, A. Sheykhi, Z. Dayyani, Critical behavior of Born-Infeld dilaton black holes. Phys. Rev. D 93, 024022 (2016)

49. J.X. Mo, G.Q. Li, X.B. Xu, Effects of power-law Maxwell field on the critical phenomena of higher dimensional dilaton black holes. Phys. Rev. D 93, 084041 (2016)

50. V. Balasubramanian, S.F. Ross, Holographic particle detection. Phys. Rev. D 61, 044007 (2000)

51. S. Ryu, T. Takayanagi, Holographic derivation of entanglement entropy from AdS/CFT. Phys. Rev. Lett. 96, 181602 (2006)

52. S. Ryu, T. Takayanagi, Aspects of holographic entanglement entropy. JHEP 0608, 045 (2006) 